\title{
Interest-driven Forwarding for Delay-tolerant Mobile Ad Hoc Networks
}

\author{
Elena Pagani \\ Computer Science Dept., Università degli Studi di Milano \\ CNR-IIT, National Research Council, Italy \\ Email: pagani@di.unimi.it
}

\author{
Gian Paolo Rossi \\ Computer Science Department \\ Università degli Studi di Milano, Italy \\ Email: rossi@di.unimi.it
}

\begin{abstract}
In the era of pervasive mobile computing, human encounters can be leveraged to enable new forms of social interactions mediated by the personal devices of individuals. In this framework, emerging needs, such as content dissemination, social discovery and question\&answering, advocate the raising of novel communication paradigms where the binding contentrecipients is not provided by the sender (in the classical IP addressing style), but directly executed by specific recipients with interest on it. This allows tagged contents to be freely advertised on the network according to a content-driven approach; human encounters drive the information towards potential recipients that extract it from the stream when content type and personal interest match. This very active research area has recently produced a few preliminary solutions to this networking problem; they inherently confine message delivery inside a specific location and/or community. This covers only a part of users needs, as emerging from everyday life experience and recent studies in human sciences.
\end{abstract}

This paper proposes a novel communication protocol, named InterestCast, or $I$ Cast, solving the problem for a wide range of social scenarios and applying to a delay tolerant ad hoc network whose nodes are the personal device of moving individuals, possibly interacting with fixed road-side devices. The protocol is able to chase users interests decoupling content tags from locations and social communities. The main advantages the proposal achieves are: it ensures remarkable performance results; it is simple and, thus, it is feasible and keeps computational and networking costs low; it preserves users privacy.

Keywords-Delay-Tolerant Networks; content-based addressing; mobile computing; human social interactions.

\section{INTRODUCTION}

Real-life encounters are the oldest form of human communication where individuals mediate the (verbal) information exchange from source(s) to recipient(s) through either single or multi-hop paths. In the era of pervasive mobile computing, human encounters have been leveraged to provide an intermittently-connected network where the delivery of delay tolerant information is mediated by the personal devices of individuals. The growing interest in human social interactions is hastening the shift from human-mediated to computermediated communications; emerging needs, such as content dissemination, social discovery and question and answering, inspire the creation of novel communication paradigms where the binding content-recipients is not provided by the sender (in the classical IP-addressing style), but directly executed by specific recipients with an interest in it. This enables unaddressed content to be freely advertised on the network with a tag describing the content type; human encounters drive the information flow towards potential recipients that extract it from the stream when content type and personal interest match. Basically, it is a new form of anycast communication that intercepts the user's interests by preserving, on the one hand, the communication resources and, on the other, the user's privacy. We call it InterestCast, or ICast for short.

This new form of social interaction requires a programming and networking platform that supports it. The design of this platform is still in its infancy. In fact, all solutions attempting to stretch the validity of the IP multicast model to the new scenario (e.g. [5], [9], [13], [21], [22]) fail because they assume that the addressing scheme is known a-priori by the sender. By contrast, here the set of recipients is unknown; its cardinality is unpredictable and changes dynamically over time as a result of mobility and temporary disconnections. This leads us to consider a delay tolerant communication scenario where the routing of messages has to face a number of challenging issues. Some preliminary results are obtained by starting from the basic assumption that the user's interests and customarily frequented locations are closely related to one another. ProfileCast [12] belongs to this category. A content generated by a node is addressed to ("is of interest for") nodes used to visit the same locations as the source. SocialCast [6] goes a little further by assuming that users with the same interests have the attitude to meet with each other more often than with other users. In ContentPlace [3], it is assumed that users belong to social communities and that communities are bound to physical places. Although it is accepted that communities may include different interests, it is assumed that there is a predominant interest inside a community and this drives communications that happen to have a community-based granularity.

All the aforementioned assumptions greatly simplify the original problem because they inherently confine message delivery within a specific location and/or community. Unfortunately, human interests are not only bound to specific locations or closely assigned to a given community. In [11] the authors observe that the correlation among all plans is not immediate, while in [15] it is shown that this correlation varies with changing scenarios. The undersound testbed application, developed in the framework of the BioNets Project [1], revealed that people are willing to exploit extemporary encounters with someone having the same interests in order to share content even if no social ties exist. By contrast, social ties may arise 
as a consequence of a shared interest.

This paper describes a new routing protocol that solves the InterestCast (ICast) problem in a delay tolerant, mobile ad hoc network of individuals, possibly interacting with fixed stations [17]. The protocol adopts a simple utility function that properly chases the user's interests independently of locations and social communities. The main advantages of this proposal are: first, the algorithm succeeds both in keeping coverage very high and in reducing the consumption of network resources (due to the generation of message copies) by nearly 37 percent on average with respect to an epidemic approach. This is achieved without observing any significant increase of delivery latency. Secondly, the algorithm adopts a very simple utility function that allows us to keep both computational and communication costs very low, i.e. the solution is viable in practice. Finally, the user's privacy is preserved because forwarding nodes do not maintain any association between user's identity and interests, and this binding is only performed by the end-system, i.e. by the user's mobile device which is considered as the extension of ourselves.

\section{System Assumptions And Problem Definition}

We assume a mobile network to be composed of $n$ nodes that communicate through wireless links. A node is either the personal device of a user, which moves with the user, or a fixed station, as in the case of a road-side gateway to/from a wired network. Thus, we are considering a hybrid urban network infrastructure [10], [20]. Throughout this paper, all nodes, whether fixed or mobile, have the same capabilities; each node operates as source, recipient and forwarder of messages with specified interests. A node stores messages in a buffer and, in the sequel, we assume that only one interest $\mathcal{I}$ is assigned to a node (this constraint is easily removed). The purpose of the protocol is to deliver a message to (approximately) all nodes matching the interest $\mathcal{I}$. Nodes beacon their onehop neighbors to advertise their interests and, as in [19], summary vectors are exchanged to prevent forwarding of duplicate messages. A content is described by a content-tag that is compared with the declared interests of nodes in order to determine its destinations. Basically, addressing is performed on a per-content basis. Content tags and interests do not have to match exactly. Folksonomic reasoning is used to match nodes interests w.r.t. content tags - for instance, as described in [14] - and when a matching is verified the message is delivered to the local recipient, or forwarded to the appropriate relay.

All nodes run the forwarding algorithm described in Section III that solves the ICast problem. The problem is defined as follows:

Definition 1 (ICast): Let $I$ be a set of recipient (endpoints) with a common interest $\mathcal{I}$. Let $U$ be the set of all recipients in the system. Then an algorithm for ICast of a message $M$ labeled with interest $\mathcal{I}$ must ensure the following properties:

- Correctness: (i) $M$ must be delivered to a subset $I^{\prime} \subseteq I$ of recipients, but (ii) no recipient in $U-I$ must deliver $M$.

- Effectiveness: the service should approximate total coverage, i.e., it should keep the cardinality of $I^{\prime}$ as close as possible to that of $I$.
- Efficiency: the service should reduce both network load and delivery latency, with respect to epidemic.

The correctness property guarantees that recipients without interest about $\mathcal{I}$ are not spammed with unwanted messages, that is, it excludes broadcast (adopted, e.g., in [20]). The effectiveness property has to do with user satisfaction and delivery fairness. In order to achieve it, other nodes may be used as relays if they increase the probability of reaching the destinations. However, the efficiency property excludes resorting to trivial solutions such as epidemic diffusion to solve the problem.

To determine the intended recipients of a ICast message, we introduce the Timed Delivery Model (TDM). In TDM, the delivery of a message is constrained within the time interval $\left(t_{1}, t_{2}\right)$, where $t_{1}$ is the time of message generation and $t_{2}$ is the message expiry time. When a message $m$ with label $\mathcal{I}$ is issued, a lifetime is also assigned to it (lifetime $=\left(t_{2}-t_{1}\right)$ ) and the intended recipients of $m$ are all nodes in $I$ who are reachable at any time during the time interval $\left(t_{1}, t_{2}\right) .{ }^{1} I$ Cast provides a best effort delivery service in $I$.

\section{FORWARDING IN INTERESTCAST}

In this Section, we describe the ICast forwarding algorithm to detect good relays for reaching nodes in the set $I$, that is, relays able to chase a given interest $\mathcal{I}$. As a consequence of having multiple recipients sharing a common interest $\mathcal{I}$, we can expect to have potentially several relays involved and that their selection should be influenced by their inclination to encounter nodes declaring $\mathcal{I}$ as an interest. Miming ranking mechanisms proposed for unicast communication, we adopt a simple utility function that is adapted to reach nodes in $I$, rather than an addressed destination. To this end, the Greedy [7] approach has shown to obtain the best performance in several different mobility scenarios [16]. By taking inspiration from the Greedy utility, we obtain ICast, whose pseudo-code is provided by Algorithm 1. In ICast, a node $p$ adjusts its utility with respect to a given interest $\mathcal{I}$ every time it encounters a node whose beacon includes $\mathcal{I}$ (lines 3-5), by incrementing a counter for $\mathcal{I}$. In the following, let us indicate with $\mathcal{U}$ the utility value obtained with this scheme.

A relevant aspect of the algorithm is the message replication mechanism (lines $13,18,20$ ). Whenever a node $p$, with no interest in $\mathcal{I}$, forwards a message $m$ to a node with higher utility (lines 15-16), $p$ delegates the other node to continue forwarding, and hence removes the copy of $m$ from its own buffer (line 18). By contrast, if $p$ forwards the message to a legitimate recipient, then $p$ maintains the message copy (line 13). In fact, its habit of encountering recipients in $I$ might be useful for delivering $m$ to others. Nodes in $I$ always maintain the message copy (line 20) and they can forward a copy to either another recipient (line 12) or a more useful relay which might be useful for delivering $m$ to other recipients. In Section IV we will show how such a computationally efficient utility satisfies the problem requirements in different conditions of user's interest distribution inside the set of nodes.

The advertisement of content on the network is worth continuing until the time validity of the tagged message

\footnotetext{
${ }^{1}$ Our model is similar to the Temporal Membership Model in [22]
} 


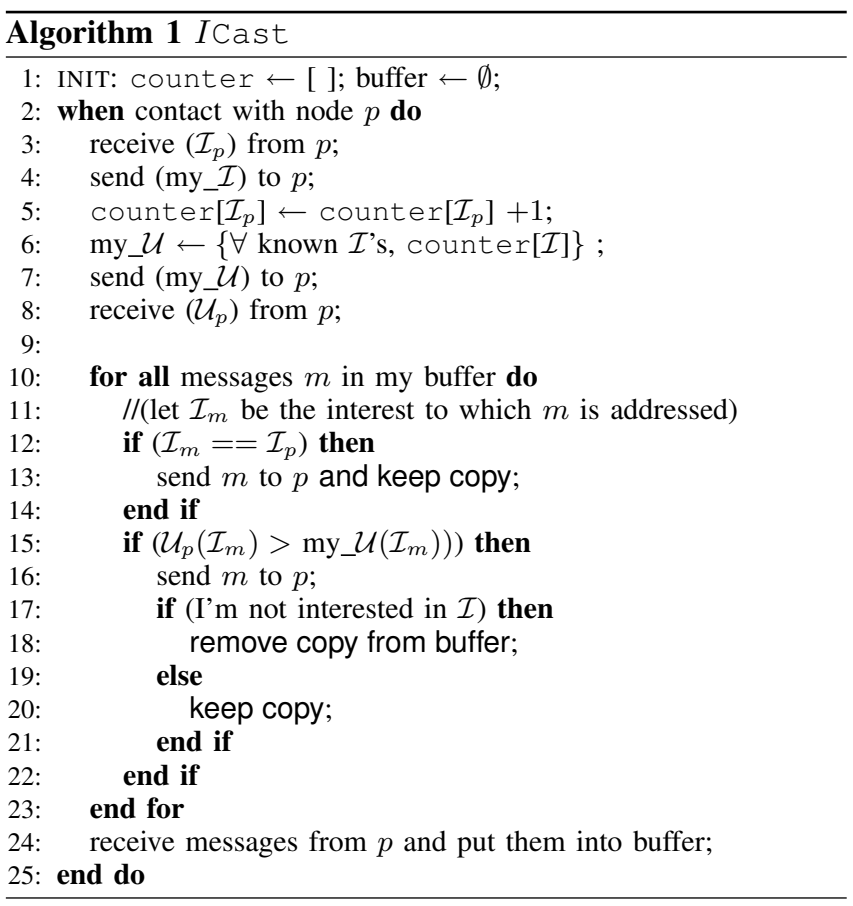

expires. This is the motivation underpinning the lifetime we introduced in section II. The time budget of $m$ is easily decremented by each single node holding it and the residual value is added to $m$ when it is forwarded to a new node. A node removes $m$ from the local buffer as soon as lifetime $=0$. Of course, the shorter the lifetime the lower the delivery probability to all recipients moving about. By contrast, a lifetime larger than the time needed to reach all recipients (i.e. the network latency time, Section IV) feeds concerns about the possible forwarding of useless message copies despite the fact that all recipients have been reached. In sec.IV-C, we show that the concerns are unmotivated; in fact, the algorithm has an interesting self-stabilization capability which ensures that all extra costs of this simple termination policy are kept very low.

\section{PERFORMANCE EVALUATION}

In this section, we present the performance evaluation of the ICast algorithm obtained by means of simulations. Simulations recreated a large variety of practical conditions (see, sec.IV-A) where sources either belong to the set of recipients or are outside of it, and recipients either belong to a given community of interests or are unequally dispersed among them. This enables us to test the algorithm behavior under far more general conditions than those assumed in previous works, such as [12] and [6]. We assume that every 30 minutes, each node generates a message labeled with interest $\mathcal{I}$. Nodes have infinite buffers, and messages have a lifetime longer than the time needed to deliver them. Message generation is stopped after 86 hours, in order to allow forwarding of the last messages to their destination by the end of the time window, whose size is 156 hours. The measured performance indexes are: coverage (percentage of recipients in $I$ that deliver the message), mean number of hops to reach a recipient, mean latency to a recipient, number of nodes involved in the forwarding of one message. All indexes are averaged over all recipients in $I$ and all sources. The mean number of hops and the number of involved forwarders are an indirect measure of ICast efficiency. ICast is evaluated against two benchmarks: the Epidemic approach and the Direct Contact approach. Epidemic [19] provides optimal coverage, paths and latency, but has the highest communication cost. Direct Contact (DirCon) is extended as follows: a source forwards a message $m$ only when it encounters an intended recipient. Each recipient receiving $m$ becomes a relay and forwards $m$ to other recipients. Direct Contact provides the minimum communication cost at the expenses of high latency and low coverage.

\section{A. Scenarios}

We consider one synthetic mobility pattern (HD5 trace) generated with the HCMM model [4], where 44 nodes move in a $1000 \times 1000 \mathrm{~m}$. area with speed in $[0.5,1.5] \mathrm{m} / \mathrm{s}$ for 156 hours; the transmission range is $10 \mathrm{~m}$. As an initial interaction matrix, we used weights derived from the number of contacts between pairs of nodes in a real trace, namely, the PMTR trace [8]. We assigned weight 0.9 to the highest number of contacts, and adopted the weight associated to half of the average number of contacts as a threshold to derive the connection matrix. No reconfiguration was performed and the remaining probability was set to 0.8 . In the HD5 trace, we adopted 5 travelers, we chose the next cell deterministically and set the rewiring probability at 0.1 .

Moreover, we analyzed the behavior of the algorithm under real mobility conditions. To this end, the PMTR trace was adopted. It involves 44 people on a campus, all equipped with wireless devices, named PMTRs (Pocket Mobility Trace Recorders), having a $10 \mathrm{~m}$. radio range [8]. We eliminated nights and weekends from the real dataset, thus producing a dataset covering 13 working days, from 8:00AM to $8: 00 \mathrm{PM}$ (for a total of 156 hours). The PMTR scenario is a difficult environment: users spend long periods in their offices, the environment is sparse, and contacts are rare [18]. We obtained long latencies and low delivery rates also when adopting an epidemic diffusion [16]. All pairs of nodes show a quite homogeneous behavior. Yet, within the considered time window, in PMTR most pairs of nodes have encounters. By contrast, in HD5 interactions between communities are supported only by a few travelers.

With the goal of reproducing possible application settings, we differentiate the scenarios in terms of distribution of $\mathcal{I}$ across social communities. We used the Louvain algorithm [2] to compute communities because it avoids grouping nodes in a giant community, and also achieves greater modularity than other algorithms in the literature. The algorithm is run for the weighted graph produced from each contact trace. In the graph, an edge exists between two nodes $n_{i}$ and $n_{j}$ if the nodes encounter at least twice; the edge weight is the reciprocal of the mean inter-contact time (ICT) between $n_{i}$ and $n_{j}$. This choice of the weights allows us to obtain communities of nodes with an habit of frequent encounters, according to the notion of social community adopted in the related work [6], [3]. With the Louvain algorithm, community detection is considered significant for modularity greater than 0.4 . We report our results in Table I; along with the modularity, the number of communities, 
TABLE I. RESULTS OF COMMUNITY DETECTION FOR THE CONSIDERED TRACES.

\begin{tabular}{|c|c|c|c|c|c|c|}
\hline scenario & modularity & \# comm. & min size & max size & mean ICT IN & mean ICT OUT \\
\hline PMTR & 0.419 & 8 & 2 & 9 & 13642 & 16785 \\
\hline HCMM_det5 & 0.488 & 7 & 3 & 12 & 3966 & 6722 \\
\hline
\end{tabular}

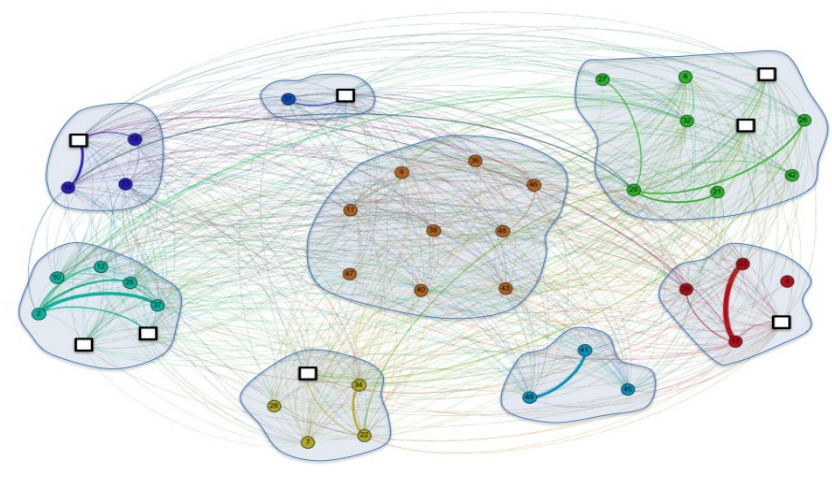

Fig. 1. Communities in PMTR with the Louvain method.

their minimum and maximum size, and the average ICT either between nodes belonging to the same community or between nodes belonging to different communities are shown. In fig.1, the PMTR graph with communities highlighted is shown, with edge thickness proportional to the weight. $^{2}$

For each trace, we built different scenarios, where nodes with an interest in $\mathcal{I}$ are unhomogeneously distributed over contact communities. Moreover, scenarios have different size. In Table II, we report the characteristics of the analyzed scenarios. For both PMTR and HD5, the following comparisons are significant:

- $\quad$ scenarios A, B, C: they differ in the size of $I$;

- $\quad$ scenarios A, D, E, F: they differ in the orthogonality between $\mathcal{I}$ and contact communities.

In PMTR, we also considered two real-life social communities, scenG involving students in the same class year, and scenH coinciding with the Computer Network research group, both distributed over 6 contact communities. In fig.1, the nodes involved in scenG are highlighted as white squares.

\section{B. Performance of ICast}

We initially compare the performance of the ICast algorithm with the two benchmarks. In fig.2, the coverage is shown. While Epidemic is almost independent of scenarios, both DirCon and ICast are somehow affected by the size of $I$ (scenarios A, B, C). The smaller the set of interested nodes, the harder it is to find the recipients. The impact of that is very different, however. While DirCon loses up to $20 \%$ of the recipients reached by Epidemic, in the worst case $I$ Cast loses just $8.5 \%$. All approaches seem quite independent of the orthogonality between $\mathcal{I}$ and the contact communities (scenarios A, D, E, F), although it is worthwhile to notice that ICast always succeeds in approximating the same coverage as Epidemic (it loses $1.5 \%$ on average).

\footnotetext{
${ }^{2}$ We emphasize that community detection is not performed by nodes, nor it is needed by the proposed algorithms.
}

TABLE II. REFERENCE SCENARIOS

\begin{tabular}{|l|l|l|}
\hline trace & scenario & characteristics \\
\hline \hline PMTR & scenA & 9 nodes in same community \\
\cline { 2 - 3 } & scenB & 5 nodes in same community \\
\cline { 2 - 3 } & scenC & 3 nodes in same community \\
\cline { 2 - 3 } & scenD & 9 nodes in 2 communities \\
\cline { 2 - 3 } & scenE & 9 nodes in 3 communities \\
\cline { 2 - 3 } & scenF & 9 nodes in 8 communities \\
\cline { 2 - 3 } & scenG & real community of 8 nodes \\
\cline { 2 - 3 } & scenH & real community of 11 nodes \\
\hline \hline HCMM_det5 & scenA & 9 nodes in same community \\
\cline { 2 - 3 } & scenB & 5 nodes in same community \\
\cline { 2 - 3 } & scenC & 3 nodes in same community \\
\cline { 2 - 3 } & scenD & 9 nodes in 2 communities \\
\cline { 2 - 3 } & scenE & 9 nodes in 3 communities \\
\cline { 2 - 3 } & scenF & 9 nodes in 6 communities \\
\hline \hline
\end{tabular}

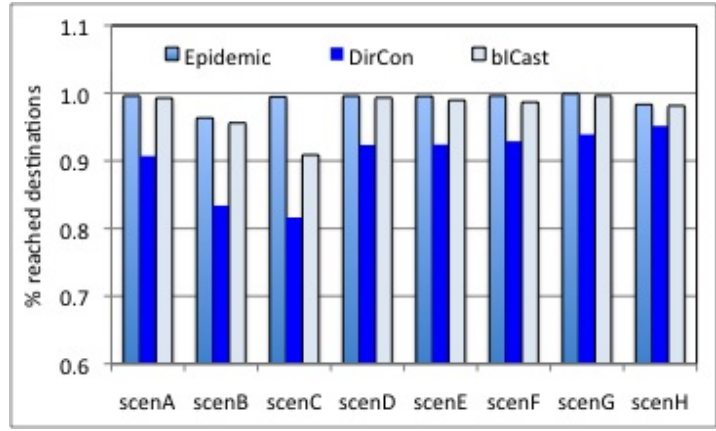

(a)

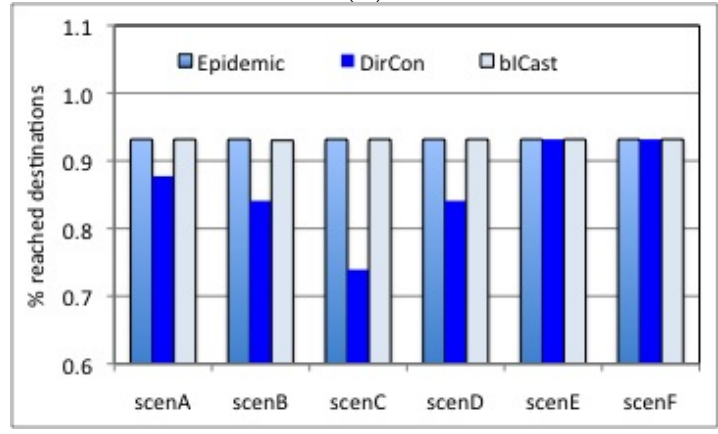

(b)

Fig. 2. Coverage for the different scenarios with (a) PMTR and (b) HD5 traces.

The most notable result is the cost paid by ICast to achieve that coverage. In fig.3, we show two performance indexes and their relationship. The first index is the decrease percentage of the number of involved forwarders with respect to Epidemic. The second index is the increase percentage of message delivery latency with respect to Epidemic. ICast is much more able than Epidemic to selectively identify the forwarders, offering high guarantees of reaching the intended recipients, though sometimes this may lead to miss the quickest path. With PMTR, in fact, by averaging the results of all scenarios, ICast involves $33.9 \%$ nodes fewer than Epidemic, 


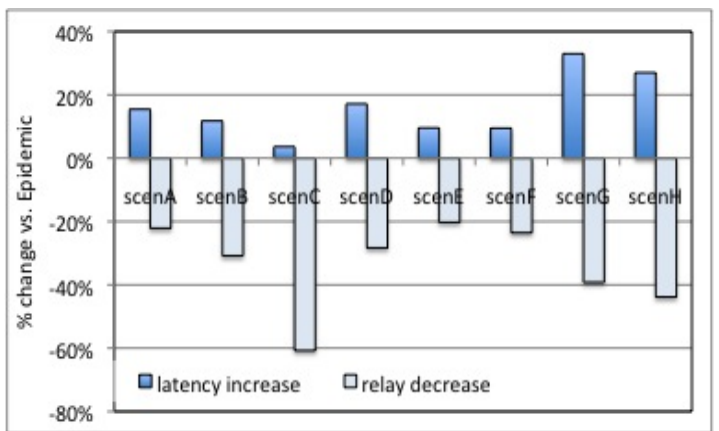

(a)

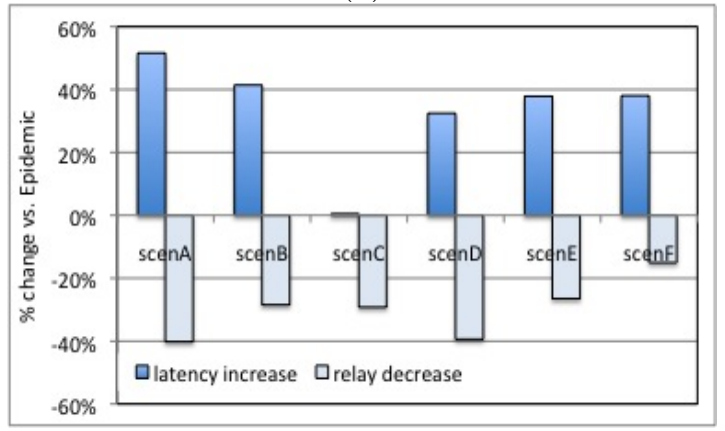

(b)

Fig. 3. Tradeoff between diffusion containment and latency decrease for the different scenarios with $(a)$ PMTR and $(b)$ HD5 traces.

and this is paid for with a latency increase of only $15.6 \%$. For the sake of comparison, the DirCon latency is two times higher than with Epidemic. It is worth observing that the remarkable reduction of involved forwarders influences positively the consumption of the overall system resources (in terms, for instance, of local memory and batteries of mobile devices). In fact, by considering the ratio between the number of radio transmissions in $I$ Cast, $T_{I}$, and in Epidemic, $T_{E}$, we obtain the value 0.73 , in scenA with PMTR trace.

With PMTR, the ability of ICast to contain the message dissemination depends on $I$ size and orthogonality of communities. ICast happens to be very effective with smaller $I$ (scenC). This means that ICast effectively selects the forwarders by focusing only on nodes that belong to the target community. Moreover, ICast does not experience changing conditions of orthogonality (scenD, scenE and scenF). Under these conditions, the algorithm shows an interesting attitude for identifying the forwarder nodes capable to act as travelers among different communities.

When HD5 is considered, the benefits are less noteworthy because of this trace's peculiar characteristic. Under these conditions, nodes are customarily confined to their communities and few travelers sporadically visit other communities. As a consequence, latency notably increases of $33.4 \%$ on average, while the reduction of number of relays is limited to $29.8 \%$. In the same synthetic scenario, the latency values in DirCon are 7-8 times higher than with Epidemic.

Finally, we observed the learning phase that the algorithm requires to acquire knowledge about the environment. This phase is very short: with PMTR - which is a difficult

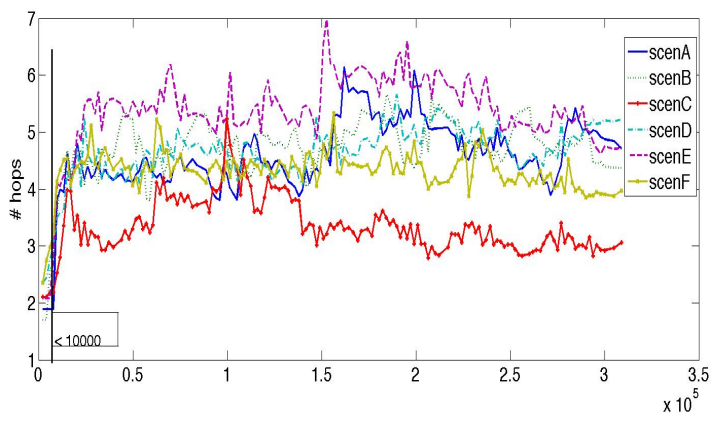

Fig. 4. Number of hops vs. time for the scenarios of the PMTR trace.

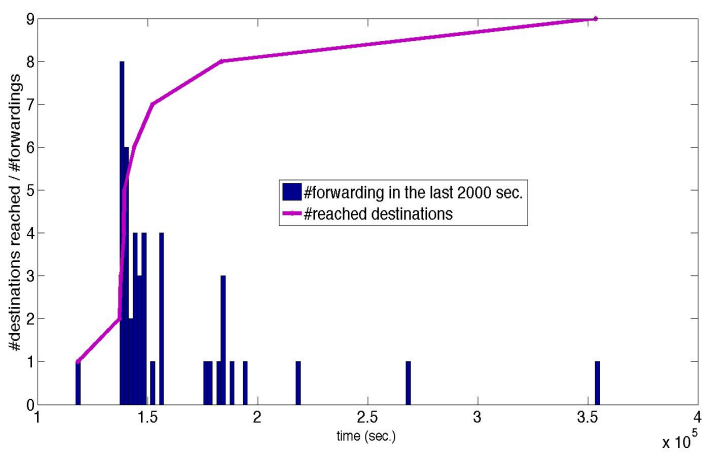

Fig. 5. Performance in terms of transmissions and reached recipients, for scenA of PMTR, and an external source.

environment [18] - ICast initially shows a behavior similar to DirCon's, because utilities are not differentiated. However, after roughly 2.5 hours, it stabilizes around the performance reported in the previous graphs. In fig.4, we report the number of hops over time for PMTR in the distinct scenarios (the line after $10000 \mathrm{~s}$. indicates the highest switch point from DirCon behavior). The paths followed by ICast are just one hop longer than those of Epidemic, on average.

\section{Stabilization of Message Transmissions}

Figure 5 shows both the number of transmissions and the cumulative number of reached recipients over time, with PMTR. The former index is measured as the number of forwarding operations in "slices" of 2000 s. of simulation: every $2000 \mathrm{~s}$. we count the number of message forwarding operations occurred from the end of the previous sample. In fig.5, results concern scenA with a source external to the social communities to which the recipients belong. Interestingly, the algorithm shows a sort of autonomic ability to self-stabilize; in fact, the number of transmissions decreases as the number of reached recipients increases. This behavior is very interesting because it means that the extra cost produced by this simple termination policy can be kept very low. This is motivated by observing that messages are forwarded from lower to higher utility nodes and are then removed from the buffers of forwarders; this gradually leads to confining message copies only in the buffers of nodes with high utility for $\mathcal{I}$, which have reduced forwarding opportunities. 


\section{CONCLUSION}

In this work, we introduce a one-to-any communication paradigm, called InterestCast, or ICast for short, in which the binding content-recipients is not provided by the sender (in the classical IP-addressing style), but directly executed by specific recipients with an interest in a tagged content. ICast provides a new form of content-driven addressing and has been explicitly devised to operate over Delay Tolerant ad hoc Networks of individuals. We describe a novel utility-based forwarding algorithm that has been proved to perfectly chase user's interest by decoupling content tags from locations or social communities, thus providing a very general solution to the problem. Furthermore, we show by means of simulations the remarkable performance and behavior of the adopted algorithm w.r.t. a classical epidemic approach.

The research area in which ICast is located is very active at the moment and may evolve in many directions. In the short term, we are focusing on three main activities. First, we are deploying an in-field experiment which will enable the understanding of existing correlations among contact/sociality/location/interest of moving people. To the best of our knowledge, this would be the first dataset publicly available to the research community and will allow to validate the ICast algorithms in a real setting. Secondly, we are paying more attention to temporal issues of the system; this activity includes (i) the modeling of the user's interest dynamics within the considered time window (to better describe the variety of conditions foreseen by the TDM, Section II), and (ii) the fine tuning of the ICast algorithms to reckon with the dynamics of the set of contacts (e.g. by introducing some aging mechanism). Finally, we are designing the node's caching and resource management system to enable the scalability of the approach. All these activities prepare the planned deployment of an ICast testbed.

\section{ACKNOWLEDGMENT}

This work was funded partially by the Italian Ministry of University and Research under the PeopleNET PRIN Project, and partially by Regione Sardegna and Regione Lombardia under the mySensing Project (BRS00015).

\section{REFERENCES}

[1] A. Bassoli, J. Brewer, K. Martin, I. Carreras, and D. Tacconi, undersound and the Above Ground, 5th Int'l Mobile Music Workshop (MMW), 2008.

[2] V.D. Blondel, J.-L. Guillaume, R. Lambiotte, and E. Lefebvre, Fast unfolding of communities in large networks, Journal of Statistical Mechanics: Theory and Experiment 2008 (10).

[3] C. Boldrini, M. Conti, and A. Passarella, Design and performance evaluation of ContentPlace, a social-aware data dissemination system for opportunistic networks, J. Comput. Netw., 54(4), 2010, 589-604.

[4] C. Boldrini, M. Conti, and A. Passarella, Users mobility models for opportunistic networks: the role of physical locations, Proc. IEEEE WRECOM, 2007, pp.1-6.

[5] M. Chuah, and Y. Xi, An Encounter-Based Multicast Scheme for Disruption Tolerant Networks, Comput. Commun., 32(16), 2009, 1742-1756.

[6] P. Costa, C. Mascolo, M. Musolesi, and G.P. Picco, Socially-aware Routing for Publish-Subscribe in Delay-tolerant Mobile Ad Hoc Networks, IEEE J. Selected Areas In Comm. (JSAC). 26(5):748-760, 2008.

[7] V. Erramilli, A. Chaintreau, M. Crovella, and C. Diot, Diversity of forwarding paths in pocket switched networks, Proc. ACM/SIGCOMM IMC, 2007, 161-174.
[8] S. Gaito, E. Pagani, and G.P. Rossi, Strangers help friends to communicate in opportunistic networks, Computer Networks J., 55(2), 2011, 374-385.

[9] W. Gao, Q. Li, B. Zhao, and G. Cao, Multicasting in delay tolerant networks: a social network perspective, Proc. 10th ACM International Symposium on Mobile ad hoc networking and computing (MobiHoc), 2009, 299-308.

[10] B. Han, P. Hui, V.A. Kumar, M.V. Marathe, G. Pei, and A. Srinivasan, Cellular traffic offloading through opportunistic communications: a case study, in Proc. CHANTS, 2010.

[11] T. Hossmann, T. Spyropoulos, and F. Legendre, Know thy neighbor: towards optimal mapping of contacts to social graphs for DTN routing, Proc. IEEE INFOCOM 2010.

[12] W.-J. Hsu, D. Dutta, and A. Helmy, Profile-Cast: Behavior-Aware Mobile Networking, SIGMOBILE Mob. Comput. Commun. Rev., 12(1), 2008, 52-54.

[13] U. Lee, S. Y. Oh, K.-W. Lee, and M. Gerla, RelayCast: Scalable Multicast Routing in Delay Tolerant Networks, Proc. ICNP 2008.

[14] G. Lo Giusto, A.J. Mashhadi, and L. Capra, Folksonomy-based Reasoning for Content Dissemination in Mobile Settings, Proc. ACM CHANTS 2010, 39-46.

[15] A. Mtibaa, M. May, and M. Ammar, On the relevance of social information to opportunistic forwarding, Int'l Symp. Modeling, Analysis and Simulation of Computer Systems, 2010, 141-150.

[16] E. Pagani and G.P. Rossi, Utility-based Forwarding: a Comparison in Different Mobility Scenarios, Proc. 3rd Int'l ACM Workshop on Mobile Opportunistic Networks (MobiOpp 2012), 29-36.

[17] T.-M. Pham, and S. Fdida, DTN support for news dissemination in an urban area, Computer Networks J., 56, 2012, 22762291.

[18] T. Phe-Neau, M. Dias de Amorim, and V. Conan, Vicinity-based DTN characterization, Proc. 3rd ACM Int'l Workshop on Mobile Opportunistic Networks (MobiOpp '12), 37-44.

[19] A. Vahdat, D. Becker, Epidemic Routing for Partially Connected Ad Hoc Networks. Technical Report CS-200006, Duke University, April 2000.

[20] J. Whitbeck, M. Dias de Amorim, Y. Lopez, J. Leguay, V. Conan, Relieving the wireless infrastructure: When opportunistic networks meet guaranteed delays, Proc. IEEE WOWMOM 2011, pp. 1-10.

[21] P. Yang, and M. C. Chuah, Context-Aware Multicast Routing Scheme for Disruption Tolerant Networks, Int. J. Ad Hoc Ubiquitous Comput. 4(5), 2009, 269-281.

[22] W. Zhao, M. Ammar and E. Zegura, Multicasting in Delay Tolerant Networks: Semantic models and Routing Algorithms, Proc. ACM SIGCOMM Workshop on Delay-tolerant networking (WDTN), 2005, 268275 . 